\title{
An Experimental Study of Stress Singularities at a Sharp Corner in a Contact Problem
}

\author{
by E.C. Miniatt, A.M. Waas and W.J. Anderson
}

ABSTRACT-The photoelastic method was used to investigate the nature of the local stress field at a sharp corner of a wedge that was compressed against a larger body. Planar wedge specimens made of photoelastic material were compressed against a half plane (larger body) of identical material at various load levels. Several wedge angles were studied. The nature of the singular stress field postulated by linear elastic analysis was verified and the strength of the singularity was obtained by plotting the variation of fringe order as a function of radial distance from the sharp corner on a logarithmic scale. The experimental results were found to be in good agreement with the theoretical predictions. The effect of interface friction and the effect of rounding of the sharp edge are briefly discussed.

\section{Introduction}

In past years, the singular nature of the stress field near a re-entrant corner defined by contact of two bodies has been accounted for by a stress-intensity factor. By using a numerical technique such as the finite-element method based on linear-elastic theory, one can obtain a detailed description of the near-field stress distribution. However, mesh refinement near the re-entrant corner will not lead to convergent stresses near the corner. Indeed, the finer the mesh, the higher the stresses become near the corner.

Many engineers in the field of engine design are somewhat skeptical about the predictions of singular stress fields. Indeed many believe that they do not exist, since problems have not occurred with current designs. One way to overcome this uncertainty is to conduct some simple model experiments. In this paper, we describe a set of experiments that are aimed at characterizing the singular stress field. One goal was too compare the experimental results with the theoretical predictions. A second was to investigate practical redesigns (e.g., chamfers, radii) that might reduce stress intensities at the contact point. The results obtained are compared with theory (Dundurs and

E.C. Miniatt is Graduate Research Assistant, A.M. Waas is Assistant Professor of Aerospace Engineering, and W.J. Anderson is Professor of Aerospace Engineering, Department of Aerospace Engineering, University of Michigan, Ann Arbor, MI 48109-2140.

Original manuscript submitted: September 22, 1989. Final manuscript received: May 10, 1990.
Lee $\left.^{1}\right)$. A discussion of the effects of frictional contact is also presented.

\section{Theory}

The configuration being studied is shown in Fig. 1. A semi-infinite body $B_{1}$ is compressed by an indenter $B_{2}$, in the form of a wedge with a sharp corner encompassing an angle $\gamma$. The half plane and the wedge have material properties $\mu_{1}, \varkappa_{1}$ and $\mu_{2}, \varkappa_{2}$ respectively. $\mu$ denotes the shear modulus while $\chi=4-3 \nu$ for plane strain with $\nu$ denoting Poisson's ratio. This problem was originally studied by Dundurs and Lee ${ }^{1}$ for the case of frictionless contact. The case with friction was later studied by Theocaris and Gdoutos. ${ }^{2}$ Comninou ${ }^{3}$ extended this study and carried out a more detailed analysis that showed the presence of logarithmic singularities. Results which are relevant to the present study are presented in this section. According to the analysis in Ref. 3, singularities in the stress field are related to the real roots of the determinant associated with the Mellin transform of the elastic field. Details of the analysis are given in Refs. 3 and 5. This determinant is expressed as

$$
D(p: \gamma, \alpha, \beta, \varrho)=8(1+p) \sin (p \pi) F(p: \gamma, \alpha, \beta, \varrho)
$$

where

$$
\begin{aligned}
& F(p ; \gamma, \alpha, \beta, \varrho)=(1+\alpha) \cos (p \pi)\left[\sin ^{2}(p \gamma)-p^{2} \sin ^{2} \gamma\right] \\
& +1 / 2(1-\alpha) \sin (p \pi)(\sin 2 p \gamma+p \sin 2 \gamma) \\
& +\varrho \sin (p \pi)\left[(1-\alpha) p(1+p) \sin ^{2} \gamma-2 \beta\left(\sin ^{2}(p \gamma)\right.\right. \\
& \text { and } \\
& \left.\left.-p^{2} \sin ^{2} \gamma\right)\right] \\
& \begin{array}{l}
\alpha=\frac{\left(\frac{\mu_{2}}{\mu_{1}}\right)\left(\varkappa_{1}+1\right)-\left(\varkappa_{2}+1\right)}{\left(\frac{\mu_{2}}{\mu_{1}}\right)\left(\varkappa_{1}+1\right)+\left(\varkappa_{2}+1\right)} \\
\beta=\frac{\left(\frac{\mu_{2}}{\mu_{1}}\right)\left(\varkappa_{1}-1\right)-\left(\varkappa_{2}-1\right)}{\left(\frac{\mu_{2}}{\mu_{1}}\right)\left(\varkappa_{1}+1\right)+\left(\varkappa_{2}+1\right)}
\end{array}
\end{aligned}
$$


Here, $\gamma$ is the wedge angle as indicated in Fig. 1 , and $\alpha$ and $\beta$ are parameters that characterize the interaction between the two elastic materials. $p$ is related to the strength of the singularities in the stresses as $r \rightarrow 0$ by

$$
\begin{aligned}
\sigma_{i j}= & O\left(r^{p-1}\right) \text { for } p \text { real and } 0 \leq p \leq 1 \\
= & O\left[r^{5-1} \cos (\eta \log r)\right] \text { or }\left[r^{-1} \sin (\eta \log r)\right] \text { for } \\
& \operatorname{complex} p=\zeta+i \eta \text { and } 0 \leq \zeta \leq 1 \\
= & O(\log r) \text { for } p=1 \text { and } \frac{\partial D}{\partial p}=0 \text { at } p=1 \\
= & O(1) \text { for no zeroes of } D \text { in } 0 \leq R e(p) \leq 1 \text { and } \\
& \frac{\partial D}{\partial p} \neq 0 \text { at } p=1
\end{aligned}
$$

For a given wedge angle, the roots of the function $F$ can be displayed on a plot of $p$ versus $\varrho$, the friction coefficient. Similarly, for a specified amount of friction (positive or negative; positive denoting slipping of the wedge relative to the half plane in the positive $x$ direction as marked in Fig. 1) the roots of $F$ can be displayed on a plot of $p$ versus $\gamma$. Consider the frictionless case $(\varrho=0)$ and let us restrict our attention to the case with power singularities characterized by $p$ real and $0 \leq p \leq 1$. Then, a typical stress component $\sigma$ can be written as

$$
\sigma=A r^{p-1}
$$

where $A$ is some function of $\theta$. Taking the logarithm of both sides it becomes evident that a linear relationship exists between $\ln (\sigma)$ and $\ln (r)$, with the slope of the straight line being related to the strength of the singularity. This observation is exploited in the present investigation to experimentally determine the value of $p$ for a range of wedge angles.

\section{Experimental Procedure}

The wedge specimens and half-plane were cut from a 0.336-in. thick plate of PSM-1 polycarbonate plastic. Each specimen and half plane were then compressed in a load frame located between the polarizer and analyzer of a Chapman Laboratories transmission polariscope. The interface was lubricated with a heavy axle grease* to model frictionless contact. The loads were measured with a 8.9-kN calibrated load cell. The loads ranged from approximately $1.6 \mathrm{kN}$ to $6.0 \mathrm{kN}$. Effort was made to obtain as symmetric a far-field load distribution as possible by fine adjustments in specimen alignment and the use of a 'tilt table' developed specifically for that purpose.

At each load increment, pictures of the near-field and far-field isochromatic fringe fields were taken. We used a Nikon F3 camera with a PN-11 extension tube and a 105-mm f/2.8 Micro-Nikkor lens. The camera was directed at the specimen through the analyzer. Full-field pictures were taken at about 1.5 meters away from the specimen. Near-field close ups were taken at about $25 \mathrm{~cm}$ from the specimen. Thus, we obtained near-field magnifications of up to 1 to 1 on the film, with which we could magnify the image to more than 16 to 1 on a film projection system.

${ }^{*}$ Kinematic viscosity of $0.135-0.165 \mathrm{~cm}^{2} / \mathrm{s}$ at $100^{\circ} \mathrm{C}$
A clear plastic ruler was placed in each picture for magnification and measurement purposes.

The specimens were loaded at about $0.89-\mathrm{kN}$ increments while careful track of fringe order was kept. The pictures were then taken and magnified, as described above, to measure the radial distance to a particular fringe order $(r)$. The radial lines were scribed at arbitrary angles to the interface. We did this to verify the separability (in $r$ and $\theta$ ) of the asymptotic stress field. Appropriate plots of fringe order against $r$ and $\hat{r}\left(\hat{r}=\frac{r}{t}\right.$, where $t$ is the thickness of the specimen) were then made and analyzed in accordance with the theory presented earlier.

The principles underlying the method of photoelasticity as a tool for studying two-dimensional stress states are well established. It suffices for us to recall that the photoelastic fringe field consists of contours, along which the maximum shear stress $\tau$ is a constant. Thus, different fringe orders correspond to different values of $\tau$.

\section{Results and Discussion}

Figure 2 shows the full-field photoelastic fringe contours of the 90-deg specimen at the highest load level. This is included to demonstrate the symmetry of the full field. Figure 3 shows the high fringe density at the reentrant corner. Figures 4 and 5 are linear-linear, log-log plots of fringe order (f.o.) versus radial distance $(r)$ and $(\hat{r})$. Note that straight lines were obtained, as predicted by the theory, by the $\ln ($ f.o.) versus $\ln (\hat{r})$. The mean values and the standard deviations of the slopes for the plots of $\ln$ (f.o.) versus $\ln (\hat{r})$, for the eight load levels of the 90 deg specimen are given in Table 1 . Note that there is no significant difference in the slopes between the two sets of data for each case, these sets corresponding to measurements taken along two different radial lines that are inclined at different angles ( $\theta$ in Table 1) to the $x$ axis. This finding held true for all of the experimental results.

Figure 6 displays a comparison of the experimentally obtained values $p$ against those predicted by theory. In this figure we have also included the now classical Williams solution ${ }^{4}$ for the free-free wedge with a vertex angle $\alpha$. This solution is useful in the present investigation as

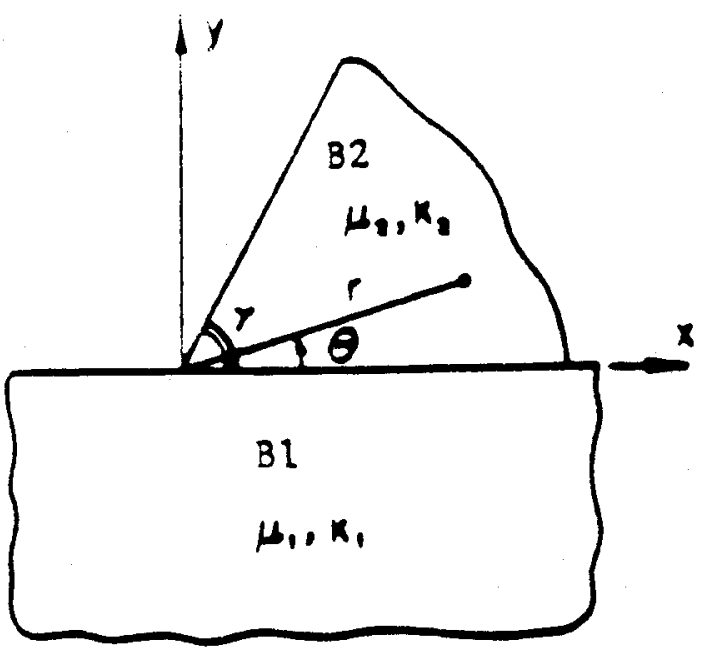

Fig. 1-Wedge in contact with half space 
we are restricting ourselves to the case of similar materials for the wedge and half plane. In this context if we view the wedge/half-plane configuration to be composed of one material (without recourse to contact), then we can specialize the Williams solution ${ }^{4}$ by substituting $\pi+\gamma$ for the vertex angle $\alpha$ in the solution presented by Williams (see Ref. 4). Also shown is the solution for frictionless contact $^{1}$ along with solutions for frictional contact ${ }^{2.3}$ generated from the aforementioned theory. As expected, the frictionless Dundurs solution is bounded by the frictional cases.

The experimentally obtained $p$ values are seen to be in good agreement with theory for wedge angles $\gamma \leq 105$

TABLE 1-MEAN AND STANDARD DEVIATION OF THE SLOPES FOR THE $\ln ($ f.o.) versus $\ln (\hat{r})$ PLOTS, MEASURED OVER EIGHT LOAD LEVELS AT TWO DIFFERENT THETA VALUES

\begin{tabular}{cr}
\hline$\theta$ (deg) & Mean \pm st. deviation \\
\hline 90 & $-0.268 \pm 0.017$ \\
70 & $-0.268 \pm 0.017$
\end{tabular}

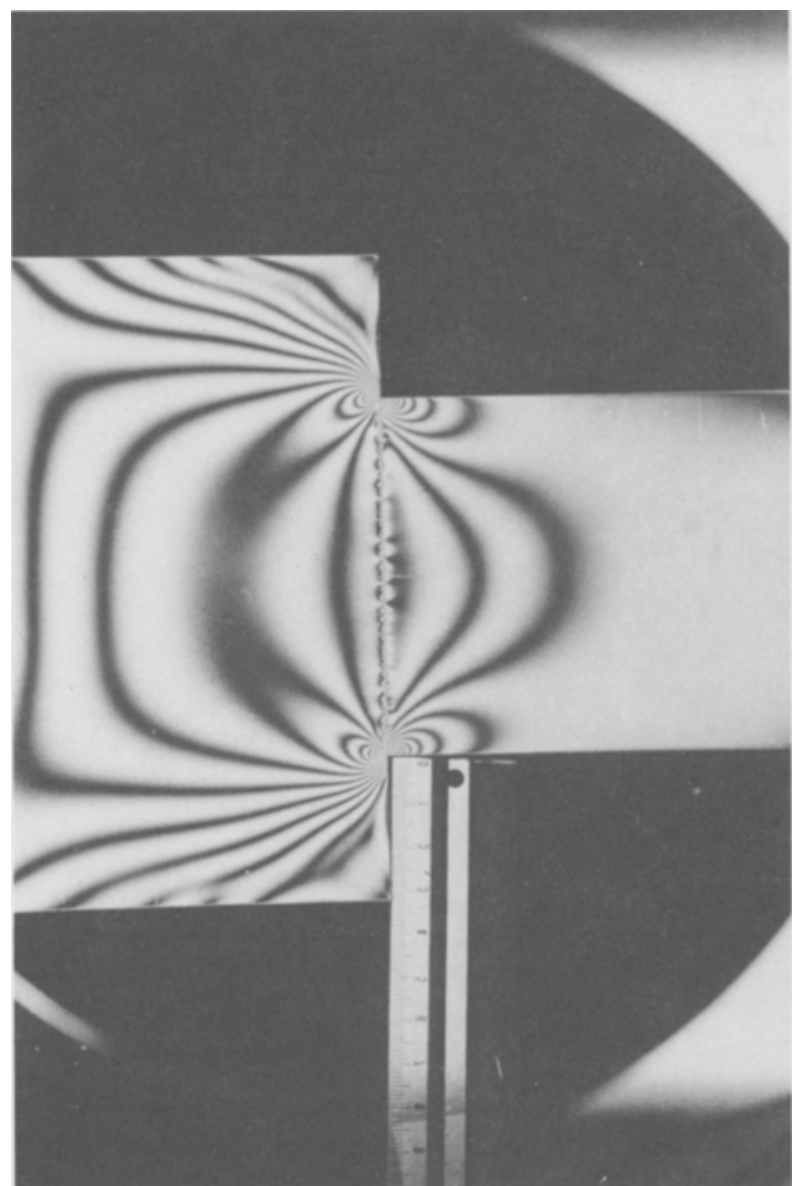

Fig. 2-Isochromatics for 90-deg wedge at highest load $(6.0 \mathrm{kN})$ deg. Further, in this range, the experimental values are bounded from above with the Dundurs and Lee solution. That is, at no time during the experiment did the experimental values of $p$ reach or exceed the theoretical prediction. For wedge angles greater than $105 \mathrm{deg}$, the $p$ values deviate away from the Dundurs and Lee solution. Consider now similar plots for the problem with friction. Then, according to the theory presented in Ref. 3, and as discussed earlier, the presence of friction at the interface, no matter how small, causes the $p$ values for any specified wedge angle to shift positively for positive slip of the wedge with respect to the half plane and negatively for negative slip. Therefore, it seems reasonable to say that a frictional interface tends to reduce the stress singularities at the corners provided that simultaneously we require positive slip of the wedge. However, as pointed out in Ref. 4, the direction of slip for a given configuration, is an unknown and cannot be predicted beforehand. In the light of this discussion, and with the reality that some amount of friction is always present in a laboratory setting, the present experimental results point towards the possibility of a reversal in the direction of slip at a wedge angle of $\approx 95 \mathrm{deg}$. This statement cannot be proven conclusively with the data we obtained. However, for $\gamma<$ $95 \mathrm{deg}$, the experimental results are nearly equal to the theoretical predictions. The deviations may be due to other factors. There is a definite effect above $\gamma=95 \mathrm{deg}$.

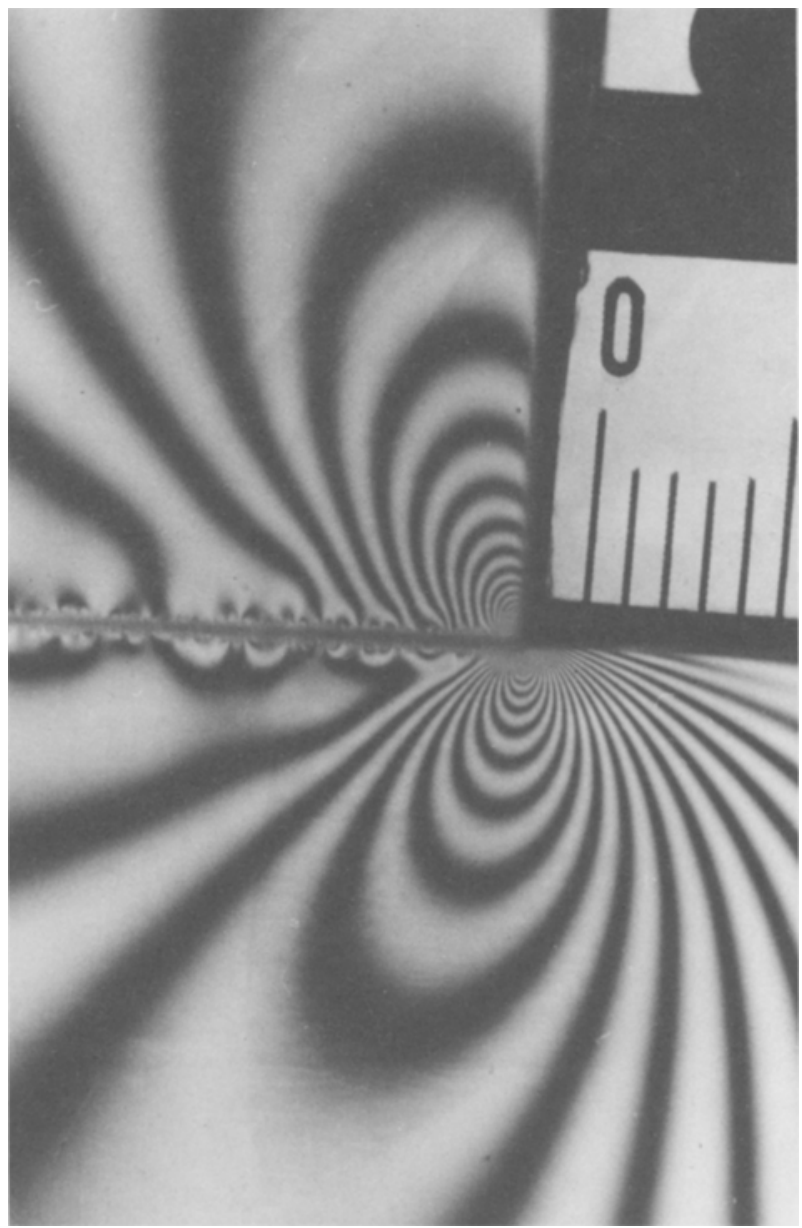

Fig. 3-Isochromatics near re-entrant corner 
For angles larger than this value, friction is seen to strongly alleviate the stresses at the corner. It must be borne in mind that the angle at which the slip direction changes is also a function of the material mismatch (characterized by $\alpha$ and $\beta$ before). However, we do not consider this case in the present investigation.

Figure 7 shows the wedge specimen with $\gamma=75 \mathrm{deg}$. Note that there is no significant pileup of fringes at the corners, characteristic of singularity points. These specimens were taken up to the highest loads but there were never more than two or three fringes in the corners. This was accompanied by a significant reduction of fringe order at the corners as compared to larger angled specimens at equitous loads. Thus, for wedge angles below $\gamma \approx 75 \mathrm{deg}$, there is no evidence of power singularities. This supports the theoretical findings for the frictionless case.

At present we are investigating the stress fields for 'wedges with rounded corners', both, convex and concave (see Fig. 7). Preliminary results for a concave corner indicate the presence of power singularities. Straight lines

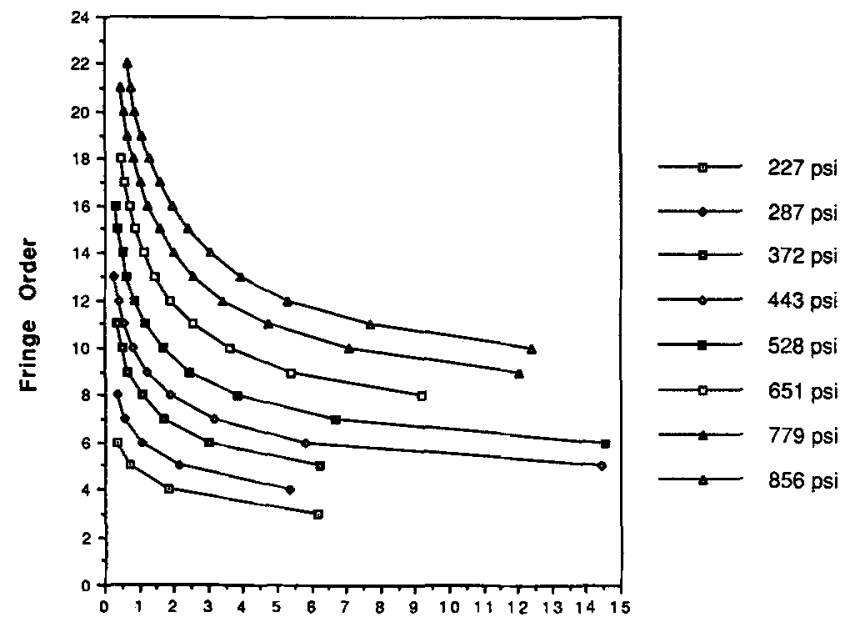

Fig. 4-Plot of fringe order against $r$ for 90-deg wedge at eight different load levels

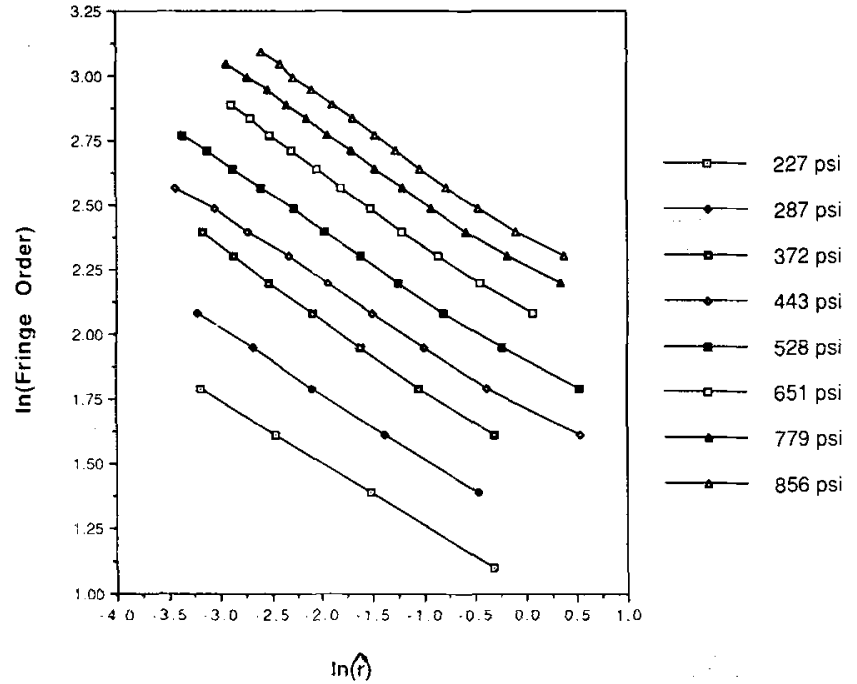

Fig. 5-Plot of In (fringe order) against $\ln (\hat{h})$ for 90 -deg wedge at eight different load levels

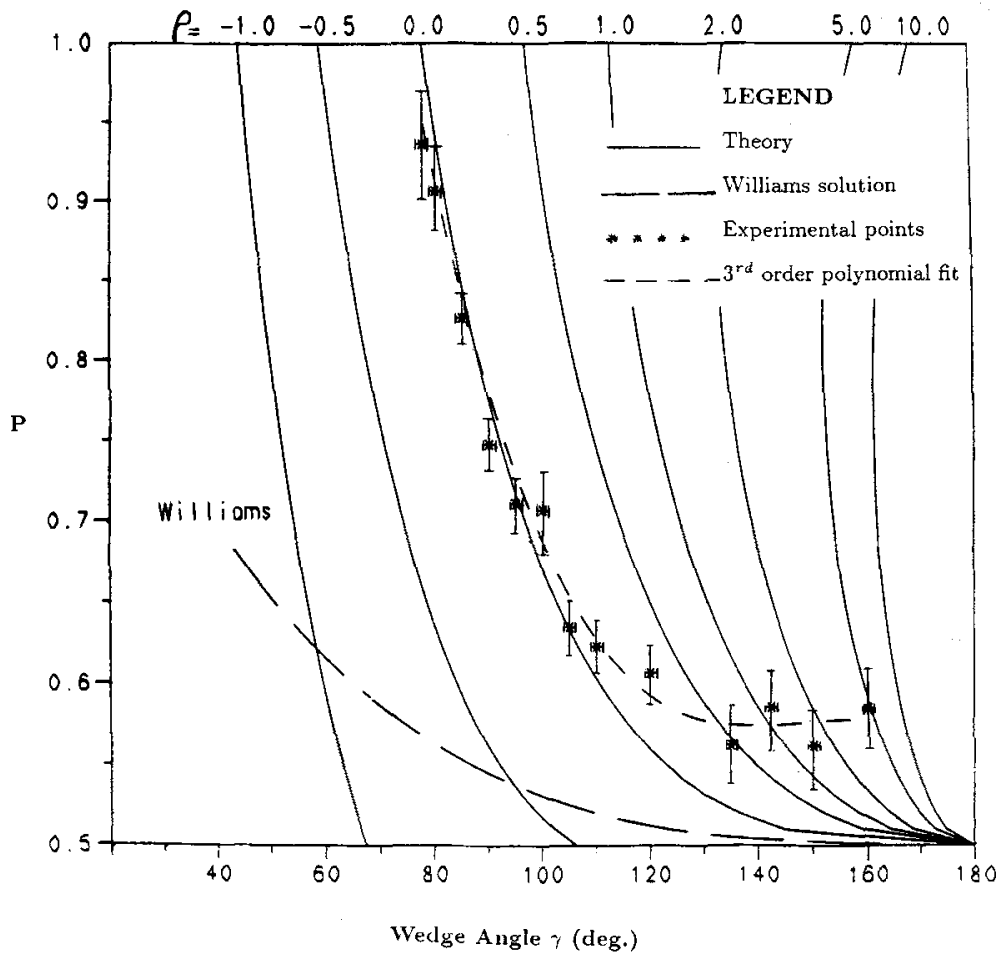

Fig. 6-Graph of $p$ versus $\gamma$ for theoretical and experimental values. The experimental data points are indicated by * 
were obtained, as before, by plotting $\ln$ (f.o.) versus $\ln (\hat{r})$, which yielded an average $p$ of $0.546 \pm .054$. We anticipated a value of $p \approx 1$, based on the premise that the concave wedge meets the half plane smoothly at an angle of $\approx 77.5 \mathrm{deg}$, deduced from theory. However, because the concave corner introduces a new length scale to the problem (the corner radius), the region in which the asymptotic stress field is dominant will now be strongly dependent on the corner radius. We are investigating this at the present time and also conducting experiments on a convex rounded corner. The possibility of logarithmic

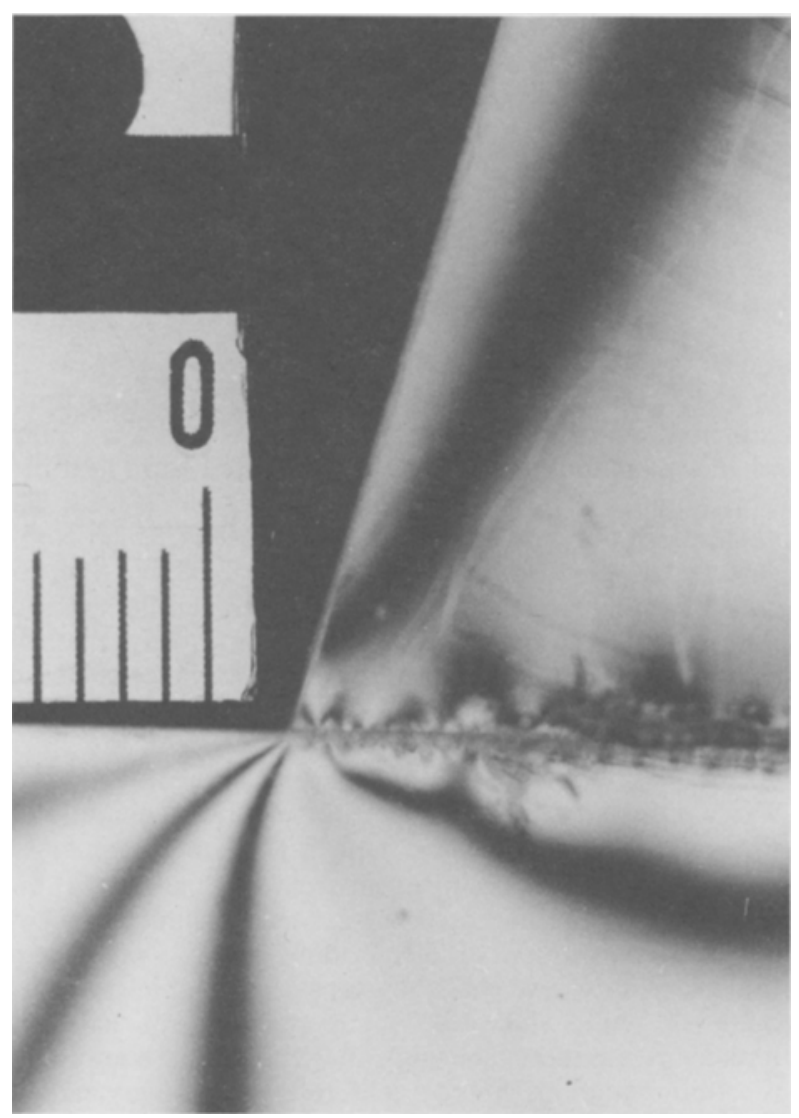

Fig. 7-Close up of 75-deg wedge at 317 psi singularities was also studied by plotting fringe order versus $\ln (\hat{r})$. This did not indicate a linear relationship.

\section{Conclusions}

In this study we have successfully used the method of photoelasticity to study the singular nature of the stress field at a sharp corner of an elastic body compressed against a larger elastic body in the form of a half plane. Some preliminary results have also been obtained for the case of a rounded corner. The good agreement between experiment and theory has demonstrated the use of the analytical work in designing configurations that alleviate the stresses due to sharp edges. The effect of friction is seen to have a favorable effect on the stress field for wedge angles $\geq 95 \mathrm{deg}$. This information cannot be obtained from theory alone since the direction of slip is configuration dependent and is an unknown in the analysis. At present, we are investigating the effect of rounded corners on the local stress field. As an improvement to the specimen preparation, a grinding process is currently being explored to improve the smoothness of the contact surfaces.

\section{Acknowledgments}

The authors acknowledge the financial support from the Ford Scientific Research Laboratories. Also, the many useful discussions and the suggestion to use photoelasticity in this investigation, by Prof. Marek Zochowski, Technical University of Warsaw, Poland, are gratefully acknowledged. The constructive comments by the reviewers have enhanced the quality of the presentation. The authors are appreciative of this gesture.

\section{References}

1. Dundurs, J. and Lee, M.-S., "Stress Concentration at a Sharp Edge in Contact Problems, " J. Elasticity, 2, 109 (1972).

2. Gdoutos, E. and Theocaris, P.S., "Stress Concentration at the Apex of a Plane Indentor Acting on an Elastic Half Plane," J. Appl. Mech., 36, 650 (1975).

3. Comninou, M., "Stress Singularity at a Sharp Edge in Contact Problems with Friction," J. Appl. Math and Phys. (ZAMP), 27, 493 (1976).

4. Williams, M.L., "Stress Singularities from Various Boundary Conditions in Angular Corners of Plates in Extension," J. Appl. Mech., 19, $526(1952)$.

5. Bogy, D.B., "Two Edge-bonded Elastic Wedges of Different Materials and Wedge Angles Under Surface Tractions," J. Appl. Mech., 38, $377(1971)$.
Fig. 8-Definition of convex and concave rounded corners
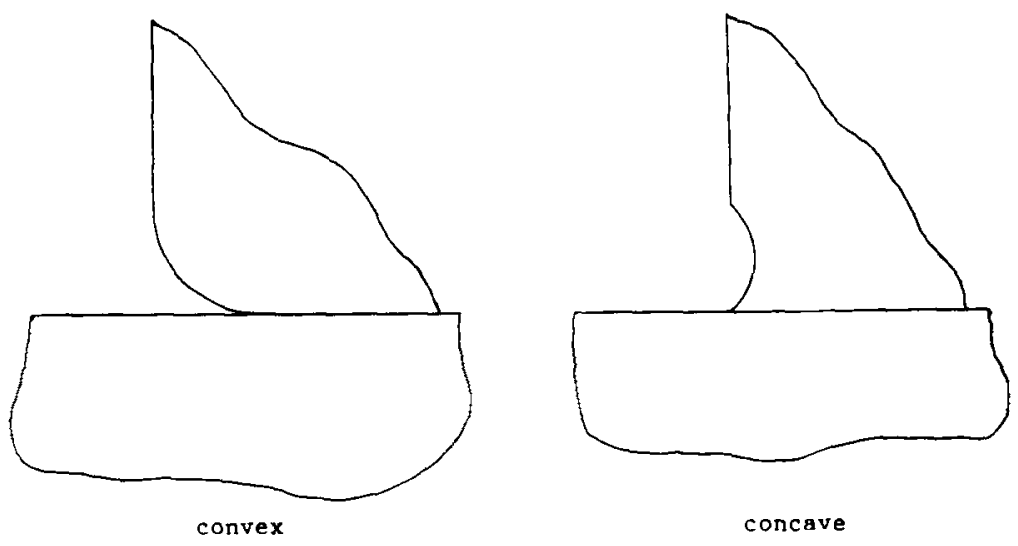